\title{
Mutations in the LKBI tumour suppressor are frequently detected in tumours from Caucasian but not Asian lung cancer patients
}

\author{
JP Koivunen 1,2, J Kim ${ }^{3,4}$, J Lee ${ }^{3,4}$, AM Rogers ${ }^{2}$, JO Park ${ }^{1,2}$, X Zhao², K Naoki', I Okamoto5 ${ }^{5}$ K Nakagawa ${ }^{5}$, \\ BY Yeap ${ }^{6}$, M Meyerson ${ }^{1,2,7,8,9}$, K-K Wong ${ }^{1,2,9}$, WG Richards ${ }^{10}$, DJ Sugarbaker ${ }^{10}$, BE Johnson ${ }^{1,2,9}$ and \\ PA Jänne ${ }^{*, 1,2,9}$
}

\begin{abstract}
'Lowe Center for Thoracic Oncology, Dana-Farber Cancer Institute, Boston, MA, USA; ${ }^{2}$ Department of Medical Oncology, Dana-Farber Cancer Institute, Haward Medical School, Boston, MA, USA; ${ }^{3}$ Department of Thoracic Surgery, Samsung Medical Center, Seoul, Korea; ${ }^{4}$ School of Medicine, Sungkyunkwan University, Seoul, Korea; ${ }^{5}$ Department of Medical Oncology, School of Medicine, Kinki University, Osaka, Japan; ${ }^{6}$ Department of Medicine, Massachusetts General Hospital, Boston, MA, USA; ${ }^{7}$ Department of Pathology, Haward Medical School, Boston, MA, USA; ${ }^{8}$ The Broad Institute of MIT and Harvard Universities, Cambridge, MA, USA; ' Department of Medicine, Brigham and Women's Hospital and Harvard Medical School, Boston, MA, USA;

${ }^{10}$ Department of Surgery, Brigham and Women's Hospital, Boston, MA, USA
\end{abstract}

\begin{abstract}
Somatic mutations of $L K B /$ tumour suppressor gene have been detected in human cancers including non-small cell lung cancer (NSCLC). The relationship between $L K B /$ mutations and clinicopathological characteristics and other common oncogene mutations in NSCLC is inadequately described. In this study we evaluated tumour specimens from 310 patients with NSCLC including those with adenocarcinoma, adenosquamous carcinoma, and squamous cell carcinoma histologies. Tumours were obtained from patients of US $(n=143)$ and Korean $(n=167)$ origin and screened for $\angle K B I, K R A S, B R A F$, and EGFR mutations using RT-PCR-based SURVEYOR-WAVE method followed by Sanger sequencing. We detected mutations in the LKB I gene in 34 tumours (I I\%). LKB I mutation frequency was higher in NSCLC tumours of US origin ( $17 \%)$ compared with $5 \%$ in NSCLCs of Korean origin $(P=0.00 I)$. They tended to occur more commonly in adenocarcinomas (13\%) than in squamous cell carcinomas $(5 \%)(P=0.066)$. $L K B \mid$ mutations associated with smoking history $(P=0.007)$ and KRAS mutations $(P=0.042)$ were almost mutually exclusive with EGFR mutations $(P=0.002)$. The outcome of stages I and II NSCLC patients treated with surgery alone did not significantly differ based on LKB I mutation status. Our study provides clinical and molecular characteristics of NSCLC, which harbour LKB I mutations. British Journal of Cancer (2008) 99, 245-252. doi: I 0.1038/sj.bjc.6604469 www.bjcancer.com
\end{abstract}

Published online I July 2008

(c) 2008 Cancer Research UK

Keywords: carcinoma; non-small cell lung; mutation; LKBI; EGFR; KRAS

Peutz-Jeghers syndrome (PJS) is caused by mutations in the LKB1 tumour suppressor gene (Hemminki et al, 1998). LKB1 is serinethreonine kinase, which has been shown to regulate cell cycle progression, apoptosis, and cell polarity (Tiainen et al, 1999). The major target of LKB1 kinase activity is thought to be AMP-activated protein kinase (AMPK). AMPK is activated under low cellular energy conditions by raising AMP levels and it phosphorylates multiple downstream targets including tuberosclerosis complex 2 gene, which represses mTOR signalling. Phosphorylation of AMPK by LKB1 is needed for full activity of AMPK and suppression of mTOR activity under low energy conditions (Shaw et al, 2004). The hallmarks of PJS include mucocutaneous pigmentation and hamartomatous polyps of the gastrointestinal tract. Patients with PJS have an increased risk of developing gastrointestinal, pancreatic, breast, gynecological, and non-small cell lung cancers (NSCLC). The overall risk for cancers is increased 5- to 12-fold in different age groups compared with the general population (Hearle et al, 2006). Somatic mutations of the

*Correspondence: Dr PA Jänne, Lowe Center for Thoracic Oncology, Dana-Farber Cancer Institute, D820A, 44 Binney Street, Boston, MA 021 I5, USA; E-mail: pjanne@partners.org

Revised 9 May 2008; accepted 28 May 2008; published online I July 2008
LKB1 tumour suppressor have rarely been found in cancers from patients who do not have PJS except for NSCLC (Avizienyte et al, 1999). Previous reports have suggested the $L K B 1$ mutation rate to be as high as $30 \%$ in NSCLC tumours and cell lines derived from patients of Caucasian origin (Carretero et al, 2004; Matsumoto et $a l, 2007)$ and to be infrequent in NSCLC patients of Asian origin (3\%) (Onozato et al, 2007). Furthermore, LKB1 mutations have been shown to be associated with adenocarcinoma histology, male gender, and smoking history (Matsumoto et al, 2007). A recent report of using a mouse model for $l k b 1$ inactivation in NSCLC has provided insights into the role of the gene in this cancer. This study showed that $l k b 1$ inactivation in combination with activating mutations of kras using inducible promoters in the lung was associated with decreased survival compared with kras mutation alone (Ji et al, 2007).

Current screening techniques for LKB1 tumour suppressor mutations rely on conventional exonic sequencing of the DNA, which can identify single base pair changes and small deletions/ insertions (Ballhausen and Gunther, 2003). The addition of multiple ligation-dependent probe amplification (MLPA), which enables detection of exonic and whole gene deletions, with exonic sequencing has increased the mutation detection rates to $80 \%$ in patients with PJS phenotype (Volikos et al, 2006). Conventional 
sequencing has also been used to detect mutations of $L K B 1$ at mRNA level and some mutations missed by sequencing at the DNA level have been discovered by mRNA-based approaches (Abed et al, 2001). However, mutant forms of LKB1 mRNAs can have a shortened half-life because of nonsense-mediated decay, which can potentially interfere with mutation detection (Abed et al, 2001).

We have recently described a rapid and sensitive enzymatic method to detect mutations in epidermal growth factor (EGFR) of DNA from fresh tissue and paraffin-embedded tissues (Janne et al, 2006). This method includes amplification of region of interest with PCR, SURVEYOR endonuclease digestion of the products, which cleaves mismatched heteroduplex DNAs, and detection of DNA fragments by sensitive high-performance liquid chromatography (HPLC) WAVE HS system. Subsequently, SURVEYORpositive specimens are fractionated in partially denaturing conditions and are Sanger-sequenced. The major advantages of SURVEYOR-WAVE method are the fast exclusion of wild-type specimens without laborious conventional sequencing and high sensitivity. The SURVEYOR-WAVE method is more sensitive than conventional sequencing as it can detect mutant DNA sequences when they are present in $1 \%$ or more of total DNA (Janne et al, 2006).

The current study was designed to analyse the incidence of $L K B 1$ mutations in NSCLC. Furthermore, we wanted to investigate the LKB1 mutational frequency in different histologies and ethnic backgrounds, and assess their correlation to smoking history, gender, stage, survival, and other oncogenic mutations in NSCLC.

\section{MATERIALS AND METHODS}

\section{Cell lines and tumour specimens}

The NSCLC cell lines A549, NCI-H1395, NCI-H1650, NCI-H1666, NCI-H1781, NCI-1975, NCI-H23, NCI-H2126, NCI-H441, NCIH820, HCC2935, HCC4006, and HCC827 were purchased from ATCC (Manassas, VA, USA). H3255, H3255GR, HCC2279, and PC-9 have been previously described (Ono et al, 2004; Tracy et al, 2004; Engelman et al, 2006). Ma1, and Ma70 are NSCLC cell lines harbouring EGFR mutations that were established at the Kinki University, Osaka, Japan. A549, NCI-H1395, NCI-H1666, NCI-H23, NCI-H2122, NCI-H2126, and NCI-H460 have previously been reported to contain $L K B 1$ mutations (Sanchez-Cespedes et al, 2002; Bamford et al, 2004; Carretero et al, 2004).

NSCLC tumours $(n=310)$ were collected from surgical resections from patients with stages I-IV NSCLC when sufficient material for RNA extraction was available. The majority of the specimens $(n=167)$ was collected at the Samsung Medical Center, Seoul, Korea. Frozen tumour tissues were collected from 809 out of 2442 patients who underwent curative resection for NSCLC from November 1995 to February 2007 at Samsung Medical Center. One or two pieces from the periphery of the tumour masses - avoiding necrotic regions - were immediately frozen at $-80^{\circ} \mathrm{C}$ until retrieved. Medical records and haematoxylin and eosin-stained slides of the specimen were reviewed by a single pathologist. Only frozen tumour tissues from adenocarcinoma or squamous cell carcinoma (according to the 2004 World Health Organization histopathological criteria) were included. Only frozen tumour tissues with a tumour cell content of more than $70 \%$ were used for further analysis. In addition, frozen tumour tissues of the following patients were excluded from the study: patients who had received preoperative neoadjuvant treatments, patients with double primary lung cancer, and patients who had undergone incomplete resections or who had not been subjected to mediastinal lymph node dissections. Selected frozen tumour tissues were used for the microdissection. Briefly, frozen tissues were lightly stained with haematoxylin-eosin to improve visualisation, and necrotic tumour tissues and intervening normal tissues were removed.
Each of the microdissected tumour tissues with a tumour cell content of more than $90 \%$ was placed in $1 \mathrm{ml}$ Easy Blue reagent of a commercially available RNA isolation kit (easy-spin ${ }^{\mathrm{TM}}$ Total RNA Extraction Kit, iNtRON Biotechnology, Gyeonggi-do, Korea), immediately homogenised by vortexing, and the total RNA was extracted. The quantity and quality of RNA were analysed using a spectrometer (Nanodrop Technologies, Rockland, DE, USA) and Agilent 2100 Bioanalyzer (Agilent RNA 6000 Nano Kit, Agilent Technologies Inc., Böblingen, Germany), respectively. Finally, 167 frozen tissues with acceptable quality of RNA (RNA Integrity Number (RIN) value over 7.0) were used for the current studies. All patients provided written informed consent.

The tumours from Caucasian patients $(n=143)$ were collected at the Brigham and Women's Hospital, Boston, MA, USA between 1991 and 1997 and have been previously published for patient characteristics and histology, and for expression profile-based clustering of the tumours (Bhattacharjee et al, 2001; Hayes et al, 2006). Frozen samples of resected lung tumours were obtained within $30 \mathrm{~min}$ of resection and subdivided into $100 \mathrm{mg}$ samples and snap frozen at $-80^{\circ} \mathrm{C}$. Each specimen was associated with an immediately adjacent sample embedded for histology in an optimal cutting temperature medium and stored at $-80^{\circ} \mathrm{C}$. Six micrometres of frozen sections of embedded samples stained with haematoxylin and eosin were used to confirm the postoperative pathological diagnosis and to estimate the cellular composition of adjacent samples. All specimens underwent pathological review by two pathologists. In all 109 tumours obtained during the same time period were excluded because they did not meet one or more of the eligibility criteria. Tissue samples were homogenised in Trizol (Life Technologies, Gaithersburg, MD, USA) and RNA was extracted and purified by using the RNeasy column purification kit (Qiagen, Chatsworth, CA, USA). Denaturing formaldehyde gel electrophoresis followed by northern blotting using a $\beta$-actin probe assessed RNA integrity. Samples were excluded if $\beta$-actin was not full length. All patients provided written informed consent. The US cohort included specimens that have previously undergone analyses and the results have been published for EGFR, KRAS, and BRAF mutations (Bhattacharjee et al, 2001; Naoki et al, 2002; Hayes et al, 2006). We reconfirmed the mutations in 30 of these specimens using the SURVEYOR-based analysis (see section SURVEYOR digestion and HPLC analysis) and found $100 \%$ concordance between the two methods (data not shown).

Cell line specimens were snap frozen and stored at $-80^{\circ} \mathrm{C}$. RNA was extracted from tumours and cell lines using Trizol (Invitrogen, Carlsbad, CA, USA), purified with Rneasy Mini Kit (Qiagen, Valencia, CA, USA) and was used for cDNA synthesis using the QuantiTect reverse transcription kit (Qiagen, Valencia, CA, USA).

\section{PCR primers and cycling conditions}

For $L K B 1$ gene analysis, PCR primers were designed to amplify the cDNA in two amplicons. PCR primers of the first amplicon were designed to hybridise to the noncoding area of the mRNA upstream of exon 1 ( $5^{\prime}$-agggaagtcggaacacaagg- $\left.3^{\prime}\right)$ and to exon 5 $\left(5^{\prime}\right.$-ccagatgtccaccttgaagc- $\left.3^{\prime}\right)$ generating a PCR product of $797 \mathrm{bp}$. The primers for the second amplicon located at exon $5\left(5^{\prime}\right.$-aacggcc tggacaccttct- $\left.3^{\prime}\right)$ and to noncoding exon 10 (5'-gaaccggcaggaagact gag- $3^{\prime}$ ) generating a product of $702 \mathrm{bp}$, which has an overlapping part with first amplicon. For SURVEYOR-WAVE analysis of $K R A S$, PCR primers $\left(5^{\prime}\right.$-ggcctgctgaaaatgactga- $3^{\prime}, 5^{\prime}$-tcctgagcctgttttgtgtct$\left.3^{\prime}\right)$ were designed to generate an amplicon of $407 \mathrm{bp}$ covering codons 12,13 and 61 , which are the codons commonly mutated in lung cancers. For SURVEYOR-WAVE mutation analysis of $B R A F$, cDNA was amplified in two overlapping amplicons $\left(5^{\prime}\right.$-aggattt cgtggtgatggag- $3^{\prime}, 5^{\prime}$-gatgacttctggtgccatcc- $3^{\prime}$, and $5^{\prime}$-gacgggactcgagt gatgat- $3^{\prime}, 5^{\prime}$-ggtatcctcgtcccaccata- $\left.3^{\prime}\right)$ covering codons 387-673. For SURVEYOR-WAVE analysis of EGFR, PCR amplification was done in a single amplicon (5'-ggagcctcttacacccagtg- $3^{\prime}$, 
$5^{\prime}$-aggtcatcaactcccaaacg- $3^{\prime}$ ), which covered exons $18-21$ of the gene. PCR amplification was done using JumpStart Taq (Sigma, St Louis, MO, USA) under the manufacturer's guidelines. A part of the specimens $(n=103)$ was previously characterised for $K R A S$, $B R A F, E G F R$ mutations using reverse transcritase (RT)-PCR and direct sequencing of the PCR products (Naoki et al, 2002; Hayes et al, 2006)

\section{SURVEYOR digestion and HPLC analysis}

SURVEYOR digestion and HPLC analysis were carried out as described previously (Janne et al, 2006). In brief, PCR products were digested in reaction mixture containing equal volumes of SURVEYOR enzyme (Transgenomics, Omaha, NE, USA) and Enhancer (Transgenomics, Omaha, NE, USA) at $42^{\circ} \mathrm{C}$ for $20 \mathrm{~min}$ followed by termination of the reaction by Stop Solution (Transgenomics, Omaha, NE, USA). Specimens were then loaded to the WAVE HS HPLC (Transgenomics, Omaha, NE, USA) at $50^{\circ} \mathrm{C}$, eluted with an increasing acetonitrile gradient, and detected by UV detector using DNA intercalating fluorescence dye (Transgenomics, Omaha, NE, USA). When cell lines known to be homozygous for specific mutation were analysed, PCR products were mixed 1:1 with PCR products of a wild-type cell line, denatured by heating, and slowly renatured to generate heteroduplexes.

\section{Sequencing and fractionation}

Specimens that showed an altered pattern on the SURVEYOR tracings were purified using QIAquick kit (Qiagen, Valencia, CA, USA) and sequenced bi-directionally by molecular biology core facility of Dana-Farber Cancer Institute. If a specimen showed an altered pattern on the SURVEYOR tracing but had a wild-type sequence by direct DNA sequencing, it underwent fractionation by WAVE HS HPLC in partially denaturing conditions. Running temperatures for specific amplicons were calculated by the Navigator Software (Transgenomics, Omaha, NE, USA). Collected fragments were amplified with PCR using the same primers as in the original amplification, purified and sequenced as previously described above.

\section{Statistical analysis}

Fisher's exact test was used to assess the association of LKB1 mutation status with other clinical, pathological, and genetic characteristics. To adjust for any difference between ethnic groups, the association between $L K B 1$ mutation rate and each characteristic was also evaluated as stratified contingency tables. If we did not reject that the odds ratios were the same across ethnic groups, we then tested whether the common odds ratios were unity based on the stratified Mantel-Haenszel estimate (Breslow and Day, 1980). Overall survival was estimated using the Kaplan-Meier method, with differences between the groups compared using the log-rank test. All $P$-values were based on a two-sided hypothesis, with $P<0.05$ considered to be statistically significant and $0.05<P<0.10$ considered to be borderline significant.

\section{RESULTS}

\section{SURVEYOR-WAVE mutation detection of $L K B 1$ tumour suppressor in NSCLC cell lines}

The impact of the stability of $L K B 1$ mRNA on detecting $L K B 1$ mutations was tested using RT-PCR with mRNA extracted from NSCLC cell lines that had previously been characterised for LKB1 mutations. These included NCI-H441 (wild type) and A549, NCIH1395, NCI-H23, and NCI-H2126 (all containing LKB1 mutations). Reverse transcriptase-PCR amplification of the whole coding region of the LKB1 mRNA showed that cell lines with nonsense (A549, NCI-H23) mutations or $1 \mathrm{bp}$ deletion (H1395) expressed mRNA with comparable size to the wild-type H441 cell line (1460 bp). H2126 cell line, which is known to have homozygous deletion of exons 4-6, expressed mRNA with substantially smaller size $(\sim 1000 \mathrm{bp})$ corresponding to deletion of $398 \mathrm{bp}$. RT - PCR revealed no major difference in $L K B 1$ mRNA expression levels between $L K B 1$ mutant or wild-type cell lines (Figure 1A).

As $L K B 1$ mutant and wild-type cell lines expressed comparable amounts of $L K B 1$ mRNA with RT - PCR, we studied the cDNA for mutations using the SURVEYOR-WAVE method. The WAVE HPLC provides a system to analyse DNA fragments smaller than $900 \mathrm{bp}$ and therefore we designed two overlapping amplicons covering exons 1-5 (797bp) and 5-9 (702 bp) to amplify the whole coding region of $L K B 1$ mRNA. PCR products of $L K B 1$ mutant cell lines were mixed $1: 1$ with the products from wild-type cell lines (H441) to generate heteroduplexes as LKB1 mutant cell lines were previously reported to be homozygous for the inactivation of the gene. SURVEYOR-WAVE analysis of the amplicon covering exons $1-5$ revealed novel peaks with the cDNA for A549, and NCI-H1395 cell lines compared with the wild type from NCI-H441 (Figure 1B). SURVEYOR-WAVE analysis of exons 5-9 showed novel peaks for the NCI-H23 cell line as well. The mutations detected with SURVEOR-WAVE were confirmed by conventional DNA sequencing and they corresponded to previous reports (Sanchez-Cespedes et al, 2002; Carretero et al, 2004). We could not detect the LKB1 mutation of $\mathrm{H} 2126$ cell line with SURVEYOR-WAVE method using a two-amplicon approach because this cell line has a homozygous deletion of exons 4-6 and the reverse primer of the first amplicon and the forward primer of the second amplicon, which lie on the deleted part of the gene (data not shown).

\section{$L K B 1$ tumour suppressor gene mutations in NSCLC tumours}

We next used the SURVEYOR-WAVE method to screen NSCLC tumour specimens $(n=310)$ for LKB1 mutations. We detected 34 LKB1 mutations (11\%) in the NSCLC tumour specimens (Table 1). The majority of the LKB1 mutations detected was deletions or insertions $(n=25,74 \%)$. The remainder was missense $(n=7,21 \%)$ and nonsense $(n=2,6 \%)$ mutations (Table 2, Figure 1C). About one-half of the deletions and insertions were small, covering $<15$ bp $(n=14,56 \%)$, whereas larger deletions $(n=11,44 \%)$ covering hundreds of base pairs were detected in the remaining specimens. Some mutational hotspots were discovered. The areas that had the same mutation in more than one tumour specimen included deletion of exon $4(n=4)$, deletion of exons 2 and 3 $(n=3), \mathrm{D} 194 \mathrm{Y} \quad(n=2)$, and P281L $(n=2)$. Interestingly, a significant portion of the mutations was located in exon 1 $(n=11,32 \%)$ but there was no area of recurrent mutations in this exon (Table 2). Of the missense mutations detected in the current study, all except R426W are in the kinase domain of the protein. Missense mutations in codons 176 and 194 have been previously characterised in PJS (Launonen, 2005). We also found four F354L alterations (data not shown) but we did not consider these as missense mutations as this alteration has previously been reported to be a rare polymorphism of the gene (Launonen et al, 2000). We did not have access to the corresponding normal tissues and therefore, we could not verify if some of the missense mutations were somatic or germline.

\section{Association of $L K B 1$ tumour suppressor mutations in NSCLC with clinicopathological characteristics}

The mutation frequency of $L K B 1$ gene was significantly higher in NSCLCs in the Caucasian cohort (Table 1). Twenty-five (17\% of specimens) of the LKB1 mutations were detected in NSCLCs 


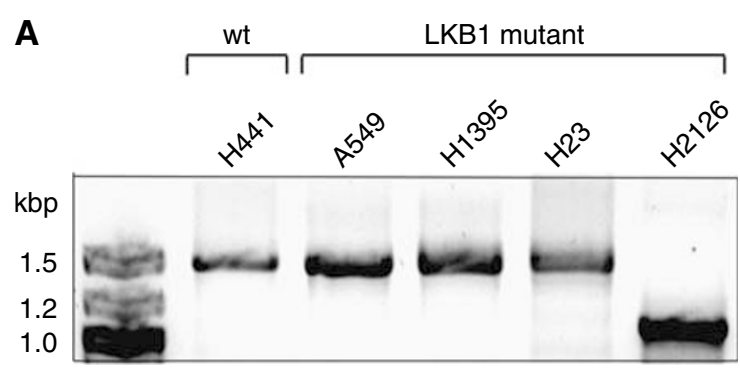

B

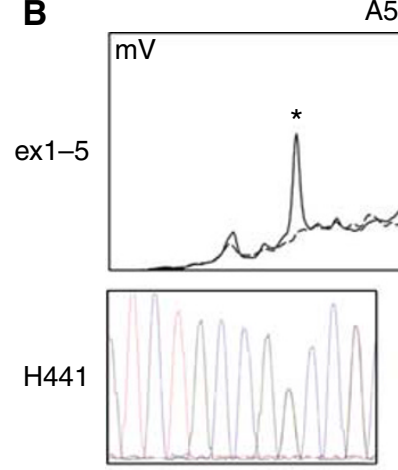

$\mathrm{A} 549+\mathrm{H} 441$
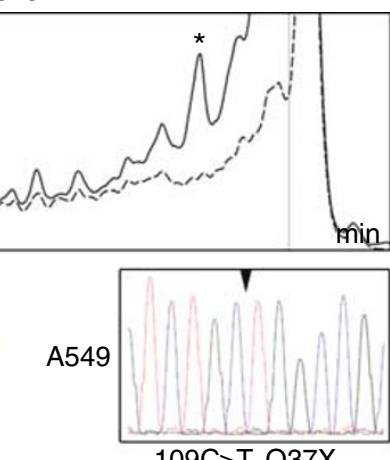

109C>T, Q37X

$\mathrm{H} 1395+\mathrm{H} 441$

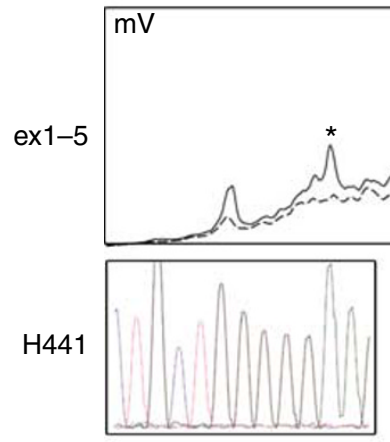

165_169delG, FS truncation

$\mathrm{H} 123+\mathrm{H} 441$

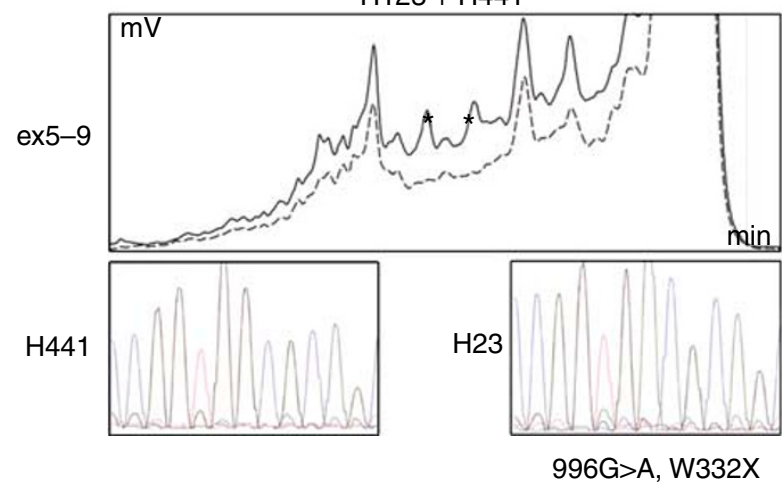

C

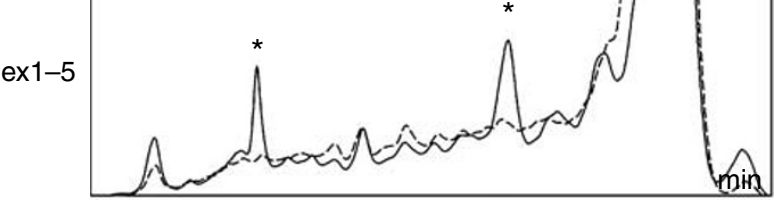

$\mathrm{H} 441$

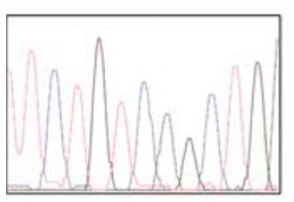

AD367

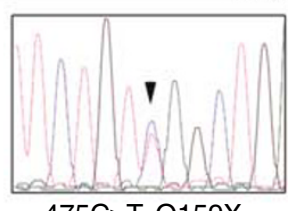

475C>T, Q159X

AD368

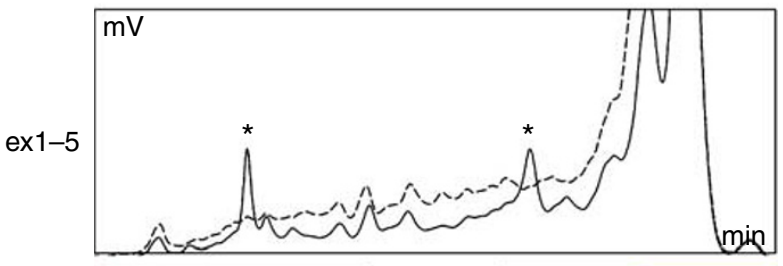

$\mathrm{H} 441$
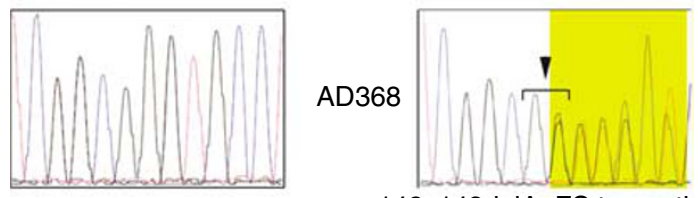

142_143delA, FS truncation

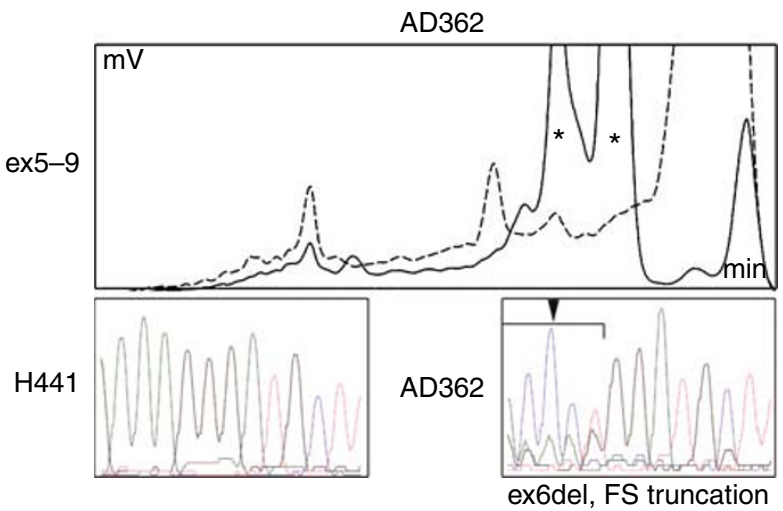

Figure I Mutation analysis of $L K B$ I gene in NSCLC cell lines and tumours. RT-PCR amplification of cDNA from $L K B \mid$ wt (H44I) and $L K B$ I mutant (A549, HI395, and H23) cell lines display the full length LKBI mRNA (I.4 kbp) while the LKBI mutant cell line, H2I 26 with a deletion of exons $4-6$ expresses a shorter mRNA ( $1.0 \mathrm{kbp})(\mathbf{A})$. HPLC tracings of SURVEYOR-WAVE mutation analysis of NSCLC cell lines A549, HI395, or H23 (continuous line), and $\mathrm{H} 44 \mathrm{I}$ (dashed line). Time in minutes is shown on the $\mathrm{X}$-axis, voltage in $\mathrm{mV}$ on the $\mathrm{Y}$-axis (B). A549 and HI395 show novel peaks (*) in the amplicon covering exons I-5 (exI-5) corresponding to I09C > T, Q37X and I65_I69delG, frameshift and truncation (FS truncation) mutations. The analysis from $\mathrm{H} 23$ demonstrates novel peaks in the amplicon covering exons 5-9 (ex5-9) corresponding to 996G > T, W332X mutation. LKB I wild-type cDNA $(\mathrm{H} 44 \mathrm{I}$ ) was added to PCR products I : I, denatured by heating and slowly renaturated to generate heteroduplexes since A549, $\mathrm{HI} 395$, and $\mathrm{H} 23$ have previously reported to be homozygous for the LKBI mutations. SURVEYOR-WAVE mutation analyses of NSCLC tumours (C). AD367 and AD368 tumours showed novel peaks in the exI-5 amplicon corresponding to 475C > T, QI59X, and I42_I43delA, FS, truncation mutations. AD362 tumour had novel peaks in ex5-9 amplicon corresponding to deletion of exon 6. Mutant sequences for AD367 and AD368 are displayed from sequences using the forward primer while mutation of the AD362 is showed with reverse primer. 
Table I Frequency of $L K B$ I mutations in NSCLC tumours and their association with clinicopathological characteristics

\begin{tabular}{|c|c|c|c|}
\hline & \multicolumn{2}{|c|}{ LKB I mutation } & \multirow[b]{2}{*}{$P$-value* } \\
\hline & + & - & \\
\hline All tumours & $34(11 \%)$ & $276(89 \%)$ & \\
\hline Age, median & 61.2 & 62.2 & \\
\hline \multicolumn{4}{|l|}{ Ethnicity } \\
\hline Caucasian cohort & $25(17 \%)$ & $118(83 \%)$ & 0.001 \\
\hline Asian cohort & $9(5 \%)$ & $158(95 \%)$ & \\
\hline \multicolumn{4}{|l|}{ Gender } \\
\hline Male & $20(11 \%)$ & 167 (89\%) & NS \\
\hline Female & $14(12 \%)$ & $107(88 \%)$ & \\
\hline \multicolumn{4}{|l|}{ Smoking } \\
\hline Never (<10 py) & $2(3 \%)$ & $70(97 \%)$ & 0.007 \\
\hline Smoker (> I0 py) & $26(14 \%)$ & $161(86 \%)$ & \\
\hline \multicolumn{4}{|l|}{ Tumour stage } \\
\hline 1 & $19(10 \%)$ & $169(90 \%)$ & NS \\
\hline$\|$ & $8(14 \%)$ & $51(86 \%)$ & \\
\hline III & $5(11 \%)$ & $42(89 \%)$ & \\
\hline IV & I (12\%) & 7 (88\%) & \\
\hline \multicolumn{4}{|l|}{ Histology } \\
\hline Adenocarcinoma & $27(13 \%)$ & I 80 (87\%) & 0.047 \\
\hline Squamous carcinoma & $5(5 \%)$ & 87 (95\%) & \\
\hline Adenosquamous & $2(22 \%)$ & 7 (78\%) & \\
\hline
\end{tabular}

*Fisher's exact test, NS = not statistically significant $(P>0.05)$.

collected from patients in the United States, whereas only nine mutations (5\% of specimens) were detected in the Korean cohort $(P=0.001)$ (Table 1$)$. The $L K B 1$ mutation rate tended to be higher in adenocarcinomas (13\%) compared with squamous cell carcinomas $(5 \%)(P=0.067)$. Differences in histological subgroups were relatively modest in the US cohort with mutations in 18 out of $94(19 \%)$ adenocarcinomas $v s 5$ out of $38(13 \%)$ in squamous cell cancers $(P=0.461)$. This is in contrast to the findings in the Asian patients where all of the LKB1 mutations were detected in adenocarcinomas (9 out of $113(8 \%)$ ) and none were detected in squamous cell cancers ( 0 out of $54(0 \%) ; P=0.032)$. Nevertheless, the higher rate of $L K B 1$ mutation in adenocarcinomas compared with squamous cell carcinomas retains the same level of statistical significance (stratified $P=0.064$ ) after adjusting for fluctuation between ethnic groups. The US cohort also included nine specimens from adenosquamous carcinomas and two out of nine (22\%) had LKB1 mutations, which is similar to the frequency in adenocarcinomas in this population (Table 1). There was no association between $L K B 1$ mutations and the clinical stage of the NSCLC patients. Kaplan-Meier survival curves of stages I and II NSCLC patients showed a tendency for shorter survival in patients with $L K B 1$ mutant tumours but this, however, did not reach statistical significance $(P=0.17)$ (Figure 2). No differences in survival were observed in patients who harboured both $L K B 1$ and KRAS mutations compared with those with KRAS or LKB1 alone but the total number of patients with both mutations who had stages I or II NSCLC was small ( $n=9$; data not shown). We detected an association of $L K B 1$ mutations with a smoking history $(P=0.007)$ and only two mutations were detected in tumours from 72 NSCLC patients who were either never or light ( $\leqslant 10$ pack years) former smokers (Table 1). After adjusting for ethnic group, the higher rate of $L K B 1$ mutation among patients with a smoking history is borderline significant (stratified $P=0.067$ ). The reduction in statistical significance is likely owing to the loss of power associated with the overall rarity of $L K B 1$ mutations among never or light former smokers. For these analyses we combined both never
Table 2 The specific $L K B /$ mutations in NSCLC tumours

\begin{tabular}{|c|c|c|c|c|c|}
\hline $\begin{array}{c}\text { Mutation } \\
\text { type }\end{array}$ & $\begin{array}{l}\text { No. } \\
\text { (\%) }\end{array}$ & Mutation & $\begin{array}{l}\text { Amino } \\
\text { acid } \\
\text { change }\end{array}$ & Exon & Histology \\
\hline \multirow[t]{7}{*}{ Missense } & $7(2 I)$ & ${ }^{\mathrm{a}} 526 \mathrm{G}>\mathrm{T}$ & DI76Y & 4 & Ad \\
\hline & & a $580 G>T$ & DI94Y & 4 & Ad \\
\hline & & $580 G>T$ & DI94Y & 4 & $\mathrm{Sq}$ \\
\hline & & $829 \mathrm{G}>\mathrm{T}$ & D277Y & 6 & AdSq \\
\hline & & ${ }^{\mathrm{a}} 842 \mathrm{C}>\mathrm{T}$ & $\mathrm{P} 28 \mathrm{IL}$ & 6 & $\mathrm{Ad}$ \\
\hline & & ${ }^{\mathrm{a}} 842 \mathrm{C}>\mathrm{T}$ & $\mathrm{P} 28 \mathrm{IL}$ & 6 & $\mathrm{Ad}$ \\
\hline & & $1276 C>T$ & R426W & 9 & Ad \\
\hline \multirow[t]{2}{*}{ Nonsense } & $2(6)$ & $206 C>A$ & $569 X$ & I & Ad \\
\hline & & $475 C>T$ & Q159X & 4 & $\mathrm{Ad}$ \\
\hline \multirow{25}{*}{$\begin{array}{l}\text { Deletion/ } \\
\text { insertion }\end{array}$} & $25(74)$ & a75_76del2\&insT & FS, truncates & I & Ad \\
\hline & & |20_|30del| | & $\mathrm{FS}$, truncates & I & $\mathrm{Ad}$ \\
\hline & & 125 I27insGG & FS, truncates & i & $\mathrm{Ad}$ \\
\hline & & 128 & $\mathrm{FS}$, truncates & I & Ad \\
\hline & & 142_143delA & $\mathrm{FS}$, truncates & I & Ad \\
\hline & & 180delC & $\mathrm{FS}$, truncates & I & Ad \\
\hline & & 209delA & $\mathrm{FS}$, truncates & 1 & $\mathrm{Ad}$ \\
\hline & & 227 228delC & $\mathrm{FS}$, truncates & i & Ad \\
\hline & & $47 \_65$ I del604 & $\mathrm{FS}$, truncates & $\mid-5$ & $\mathrm{Sq}$ \\
\hline & & 153_536del384 & $\mathrm{FS}$, truncates & $1-4$ & AdSq \\
\hline & & a exon 2-3del & Truncates & $2-3$ & $\mathrm{Sq}$ \\
\hline & & a exon 2-3del & Truncates & $2-3$ & $\mathrm{Ad}$ \\
\hline & & exon 2-3del & Truncates & $2-3$ & Ad \\
\hline & & exon 2-4del & FS, truncates & $2-4$ & $\mathrm{Sq}$ \\
\hline & & 464_465del2insTTTGCT & $\mathrm{FS}$, truncates & $3-4$ & Sq \\
\hline & & 562_563delG & $\mathrm{FS}$, truncates & 4 & Ad \\
\hline & & ${ }^{\mathrm{a}}$ exon 4del & $\mathrm{FS}$, truncates & 4 & Ad \\
\hline & & exon 4del & $\mathrm{FS}$, truncates & 4 & $\mathrm{Ad}$ \\
\hline & & exon 4del & $\mathrm{FS}$, truncates & 4 & Ad \\
\hline & & exon 4del & $\mathrm{FS}$, truncates & 4 & $\mathrm{Ad}$ \\
\hline & & 610_623dell4 & $\mathrm{FS}$, truncates & 5 & Ad \\
\hline & & a 837 844delC & $\mathrm{FS}$, truncates & 6 & $\mathrm{Ad}$ \\
\hline & & 837_844insC & $\mathrm{FS}$, truncates & 6 & Ad \\
\hline & & exon 6del & $\mathrm{FS}$, truncates & 6 & $\mathrm{Ad}$ \\
\hline & & 1038_1040insG & $\mathrm{FS}$, truncates & 8 & Ad \\
\hline
\end{tabular}

$\mathrm{Ad}=$ Adenocarcinoma; $\mathrm{AdSq}=$ Adenosquamous carcinoma; $\mathrm{Sq}=$ Squamous cell carcinoma; . 'These mutations were detected in Korean NSCLC patients.

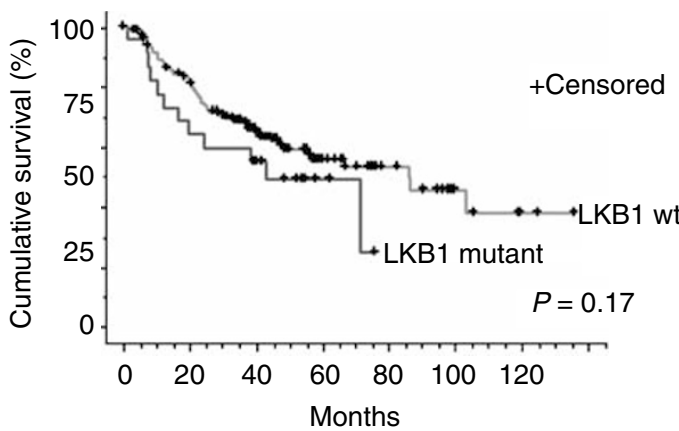

Figure 2 Kaplan-Meyer survival curves of stage I and II NSCLC patients with LKBI wildtype (red line, $n=198$ ) vs LKBI mutant (blue line, $n=23$ ) tumours.

smokers and light ( $\leqslant 10$ pack years) smokers as the frequency of mutations in other oncogenes such as EGFR is similar in these two patient groups (Pham et al, 2006). There were no correlations between LKB1 mutations and gender or age of a patient.

\section{Association of $L K B 1$ mutations with $K$-Ras, B-Raf, and EGFR mutations in NSCLC}

Previous reports have suggested that in NSCLC cell lines, LKB1 mutations often occur concurrently with $K R A S$ or $B R A F$ mutations 
(Sanchez-Cespedes et al, 2002; Carretero et al, 2004). Furthermore, EGFR mutations are often mutually exclusive with KRAS mutations in NSCLC (Kosaka et al, 2004; Marchetti et al, 2005). We used combined data from previous papers (Sanchez-Cespedes et al, 2002; Carretero et al, 2004) and from Sanger institute's databases (Bamford et al, 2004) to analyse association of LKB1 mutations with mutations of KRAS, BRAF, and EGFR. Analysis of LKB1 mutation harbouring NSCLC cell lines (A-427, A549, NCI-H1395, NCI-H1666, NCI-H2122, NCI-H2126, NCI-H23, and NCI-H460) showed that five of the cell lines $(63 \%)$ had concurrent $L K B 1$ and KRAS mutations, two (25\%) had concurrent LKB1 and BRAF mutations, and only one (13\%) had neither KRAS nor BRAF mutations. None of these cell lines had EGFR mutations.

As our findings in NSCLC cell lines suggested concurrency of $K R A S$ or $B R A F$ and mutual exclusiveness of EGFR mutations with LKB1 mutations, we analysed the mutational status of these genes in our primary NSCLC tumour specimens. KRAS mutations were detected in 49 (16\% in the whole tumour set, $25 \%$ in Caucasian and $8 \%$ in Asian specimens) tumour specimens with 10 (20\% of KRAS mutants) of these occurring concurrently with an $L K B 1$ mutation $(P=0.042)$ (Table 3$)$. Four BRAF mutations $(1 \%)$ were found in the tumour set (G465V, N581S, L596R, and T599I) and one of these (N581S) occurred concurrently with $L K B 1$ mutation $(P=0.373)$. Seventy tumours $(23 \%$ in the whole tumour set, $9 \%$ in Caucasian, $34 \%$ in Asian specimens) had EGFR kinase domain mutations with only one of them occurring concurrently with an $L K B 1$ mutation $(P=0.002)$. The tumour with a concurrent EGFR and LKB1 mutation had a missense mutation of $L K B 1$ outside the kinase domain (R426W). No germ line DNA was available from this patient. However, a recent report has suggested that R426W is in fact a rare polymorphism of the gene (Onozato et al, 2007). Taken

Table 3 Association of $L K B \mid$ mutations with KRAS, BRAF, and EGFR mutations in NSCLC tumours

\begin{tabular}{|c|c|c|c|c|}
\hline & & \multicolumn{2}{|c|}{ LKBI mutation } & \multirow[b]{2}{*}{$P$-value* } \\
\hline & & + & - & \\
\hline \multirow[t]{2}{*}{ EGFR mutation } & + & I & 69 & \multirow[t]{2}{*}{0.002} \\
\hline & - & 33 & 207 & \\
\hline \multirow[t]{2}{*}{ K-Ras mutation } & + & 10 & 39 & \multirow[t]{2}{*}{0.042} \\
\hline & - & 24 & 237 & \\
\hline \multirow[t]{2}{*}{ B-Raf mutation } & + & । & 3 & \multirow[t]{2}{*}{0.373} \\
\hline & - & 33 & 273 & \\
\hline
\end{tabular}

*Fisher's exact test.

Table 4 LKBI genotypes of NSCLC cell lines with EGFR or ERBB2 mutations

\begin{tabular}{lccc}
\hline Cell line & EGFR genotype & HER2 genotype & LKBI genotype \\
\hline HI650 & E746_A750del & $W t$ & $W t$ \\
HI78I & Wt & G776V, Cins & $W t$ \\
HI975 & L858R, T790M & $W t$ & $W t$ \\
H3255 & L858R & $W t$ & $W t$ \\
H3255GR & L858R, T790M & $W t$ & $W t$ \\
H820 & L747_L75I Idel, T790M & $W t$ & $W t$ \\
HCC2279 & E746_A750del & $W t$ & $W t$ \\
HCC2935 & E746_T75Idel, S7521 & $W t$ & $W t$ \\
HCC4006 & L747_E749del, A750P & $W t$ & $W t$ \\
HCC827 & E746_A750del & $W t$ & $W t$ \\
Ma-I & E746_A750del & $W t$ & $W t$ \\
Ma-70 & L858R & $W t$ & $W t$ \\
PC-9 & E746_A750del & $W t$ & $W t$ \\
\hline
\end{tabular}

Wt $=$ wild type. together our findings suggest that unlike KRAS, mutations in EGFR and $L K B 1$ are mutually exclusive in NSCLC.

Previous reports (Sanchez-Cespedes et al, 2002; Carretero et al, 2004) and Cancer Genome Project by Sanger Institute (Bamford et al, 2004) have extensively characterised $L K B 1$ mutations in NSCLC cell lines with KRAS and BRAF mutations, but $L K B 1$ status of EGFR mutant NSCLC cell lines has not been extensively analysed. Therefore, we analysed the $L K B 1$ genotype of NSCLC cell lines with known EGFR or ERBB2 mutations. Twelve EGFR mutant and one $E R B B 2$ mutant cell lines were analysed for $L K B 1$ genotype. No LKB1 mutations were detected in these cell lines (Table 4).

\section{DISCUSSION}

The present study characterised $L K B 1$ mutation frequency in NSCLC using one of the largest tumour sets to date $(n=310)$. Our study analysed tumours from different histologies and of both a US and Korean origin to determine potential histological and ethnic variation in $L K B 1$ mutational frequency. The large size of our study enabled us to study associations of $L K B 1$ mutations with clinocopathological factors, which have been incompletely characterised in previous studies (Sanchez-Cespedes et al, 2002; Carretero et al, 2004; Fernandez et al, 2004; Matsumoto et al, 2007; Onozato et al, 2007). In addition, we used a modification of a sensitive mutation screening technique that we have previously developed to facilitate the rapid detection of $L K B 1$ mutations (Janne et al, 2006).

Findings from our study confirm the high frequency of $L K B 1$ mutations in NSCLC (11\%), which in contrast, are rare $(0-4 \%)$ in other common solid malignancies (Avizienyte et al, 1998, 1999). The reason behind these observations is presently unknown but might reflect the differences in carcinogen exposure in the lungs compared with other tissues. In support of this hypothesis, we find that $L K B 1$ mutations are significantly $(P=0.007)$ more common in smokers than in never or light ( $\leqslant 10$ pack years) cigarette smokers (Table 1). Male PJS patients (age $\geqslant 50$ years) have an increased risk of developing lung cancer compared with the general population but the relationship of smoking and the increased risk of lung cancer in PJS is unknown (Hearle et al, 2006). Interestingly, the $L K B 1$ mutation spectrum found in the current study is very similar to those previously published for PJS (deletions 34\%, insertions $15 \%$, splice site mutations $14 \%$, missense mutations $21 \%$, and nonsense mutations 12\%) (Launonen, 2005) and, as in PJS, no clear mutational hotspots were detected.

Our study also demonstrated that the $L K B 1$ mutation frequency was significantly higher in cancers derived from a US population compared with those found in Korean patients (17 vs 5\%; $P=0.001)$. These differences also track with cigarette smoking, as the number of never/light former smokers was much higher in the Korean cohort compared with the US cohort of patients (38vs $13 \%)$. Similarly, a recent study of 100 Japanese NSCLCs found that only $3 \%$ contained an LKB1 mutation (Onozato et al, 2007). These findings are in contrast to EGFR mutations, which are more frequently detected in tumours from never/light cigarette smokers and from Asian patients (Janne and Johnson, 2006). Our studies further highlight ethnic and environmental differences in the origins of NSCLC.

Given the differences in $L K B 1$ mutation frequencies in smokers vs never/light smokers and in the US compared with Korean patients, we further determined whether these were also associated with other oncogene mutations known to vary in these subgroups of patients. Consistent with prior studies we found a significant association with concurrent KRAS mutations, which are common in smokers (Ahrendt et al, 2001), in one out of three of NSCLC with $L K B 1$ mutations (Table 3 ). In contrast, there was a significant inverse relationship of $L K B 1$ mutations with EGFR mutations in both NSCLC tumours and cell lines, which has not previously been 
described (Tables 3 and 4). These differences may relate to the biological role of LKB1 in lung cancer. It is possible that in EGFR mutant lung cancers there is already maximal activation of the $\mathrm{PI} 3 \mathrm{~K} / \mathrm{Akt} / \mathrm{mTOR}$ signalling pathway and thus an LKB1 mutation may not be required to further potentiate this signalling pathway. In contrast in KRAS mutant cancers, a concurrent $L K B 1$ mutation may be required to enhance mTOR activation. Mice with concurrent KRAS mutations and LKB1 inactivation have more aggressive tumours and a shorter survival than those with only KRAS mutant cancers (Ji et al, 2007). In our study, we were not able to detect a significant survival difference for patients whose tumours contained LKB1 mutations alone or concurrently with KRAS mutations (data not shown) likely because of the limited number of tumour specimens. Additional studies are needed to clarify the prognostic impact of $L K B 1$ mutations in humans with NSCLC. In the present study $\sim 2$ out of 3 of $L K B 1$ mutant tumours were KRAS wild type (Table 3 ). One possibility is that such tumours contain a concurrent mutation in another oncogene that activates the same signalling pathway as KRAS. For this reason, we examined our tumours for BRAF mutations, which are found in 1$2 \%$ of NSCLC (Naoki et al, 2002). We detected a concurrent LKB1 mutation in one of the four BRAF mutant tumours (Table 3). This tumour was wild type for KRAS (data not shown). In addition, some of the BRAF mutant NSCLC cell lines (NCI-H1395, G469A; NCIH1666, G466V) also contain a concurrent LKB1 mutation (SanchezCespedes et al, 2002; Bamford et al, 2004; Carretero et al, 2004). Future studies will help further clarify whether $L K B 1$ mutations occur concurrently with other genomic alterations in NSCLC and the impact of this on patient outcome.

Our study employed a mutation scanning technology to screen for $L K B 1$ mutations at the cDNA level (Janne et al, 2006). This was advantageous as the entire coding region of $L K B 1$ could be rapidly screened for a mutation using just two overlapping cDNA fragments. $L K B 1$ is a challenging gene to analyse at the genomic DNA level because of its high guanine-cytosine content. In addition, as many of the LKB1 mutations are small deletions (Table 2) or involve deletions of entire exons, these would be missed using exon-specific genome sequencing methods. Our method, however, does have limitations as it would miss deletions at the site of PCR primers, whole gene deletions, or deletions within the promoter region all of which have been infrequently detected in PJS (Volikos et al, 2006). Thus our studies may underestimate the true LKB1 mutation frequency in NSCLC. In addition, our method is limited to the analysis of fresh tumour specimens, which are available only from the minority of NSCLC patients. Furthermore, as techniques isolating mRNA from formalin-fixed paraffin-embedded tumour specimens continue to improve, this rapid mutation scanning technique can be used to analyse broader populations of tumours from NSCLC patients. Future studies may need to employ a combination of LKB1 mutation detection methodologies including the current method, MLPA and direct sequencing.

\section{ACKNOWLEDGEMENTS}

This study is supported by grants from the National Institutes of Health 1RO1CA114465-01 (BYY, BEJ, and PAJ), the National Cancer Institute Lung SPORE P20CA90578-02 (BYY and BEJ), American Cancer Society RSG-06-102-01-CCE (BYY and PAJ), Finnish Medical Foundation (JPK), Finnish Cultural Foundation (JPK), and Academy of Finland (JPK). PAJ, MM, and BEJ are part of a pending patent application on EGFR mutations.

\section{REFERENCES}

Abed AA, Gunther K, Kraus C, Hohenberger W, Ballhausen WG (2001) Mutation screening at the RNA level of the STK11/LKB1 gene in PeutzJeghers syndrome reveals complex splicing abnormalities and a novel mRNA isoform (STK11 c.597(insertion mark)598insIVS4). Hum Mutat 18: $397-410$

Ahrendt SA, Decker PA, Alawi EA, Zhu Yr YR, Sanchez-Cespedes M, Yang SC, Haasler GB, Kajdacsy-Balla A, Demeure MJ, Sidransky D (2001) Cigarette smoking is strongly associated with mutation of the K-ras gene in patients with primary adenocarcinoma of the lung. Cancer 92: $1525-1530$

Avizienyte E, Loukola A, Roth S, Hemminki A, Tarkkanen M, Salovaara R, Arola J, Butzow R, Husgafvel-Pursiainen K, Kokkola A, Jarvinen H, Aaltonen LA (1999) LKB1 somatic mutations in sporadic tumors. Am J Pathol 154: 677-681

Avizienyte E, Roth S, Loukola A, Hemminki A, Lothe RA, Stenwig AE, Fossa SD, Salovaara R, Aaltonen LA (1998) Somatic mutations in LKB1 are rare in sporadic colorectal and testicular tumors. Cancer Res 58: 2087-2090

Ballhausen WG, Gunther K (2003) Genetic screening for Peutz-Jeghers syndrome. Expert Rev Mol Diagn 3: 471-479

Bamford S, Dawson E, Forbes S, Clements J, Pettett R, Dogan A, Flanagan A, Teague J, Futreal PA, Stratton MR, Wooster R (2004) The COSMIC (Catalogue of Somatic Mutations in Cancer) database and website. $\mathrm{Br} J$ Cancer 91: $355-358$

Bhattacharjee A, Richards WG, Staunton J, Li C, Monti S, Vasa P, Ladd C, Beheshti J, Bueno R, Gillette M, Loda M, Weber G, Mark EJ, Lander ES, Wong W, Johnson BE, Golub TR, Sugarbaker DJ, Meyerson M (2001) Classification of human lung carcinomas by mRNA expression profiling reveals distinct adenocarcinoma subclasses. Proc Natl Acad Sci USA 98: $13790-13795$

Breslow NE, Day NE, International Agency for Research on Cancer (1980) Statistical methods in cancer research. Vol. 1. Analysis of case-control studies. IARC scientific publications; no. 32. International Agency for Research on Cancer: Lyon
Carretero J, Medina PP, Pio R, Montuenga LM, Sanchez-Cespedes M (2004) Novel and natural knockout lung cancer cell lines for the LKB1/STK11 tumor suppressor gene. Oncogene 23: 4037-4040

Engelman JA, Mukohara T, Zejnullahu K, Lifshits E, Borras AM, Gale CM, Naumov GN, Yeap BY, Jarrell E, Sun J, Tracy S, Zhao X, Heymach JV, Johnson BE, Cantley LC, Janne PA (2006) Allelic dilution obscures detection of a biologically significant resistance mutation in EGFRamplified lung cancer. J Clin Invest 116: 2695-2706

Fernandez P, Carretero J, Medina PP, Jimenez AI, Rodriguez-Perales S, Paz MF, Cigudosa JC, Esteller M, Lombardia L, Morente M, Sanchez-Verde L, Sotelo T, Sanchez-Cespedes M (2004) Distinctive gene expression of human lung adenocarcinomas carrying LKB1 mutations. Oncogene 23: $5084-5091$

Hayes DN, Monti S, Parmigiani G, Gilks CB, Naoki K, Bhattacharjee A, Socinski MA, Perou C, Meyerson M (2006) Gene expression profiling reveals reproducible human lung adenocarcinoma subtypes in multiple independent patient cohorts. J Clin Oncol 24: 5079-5090

Hearle N, Schumacher V, Menko FH, Olschwang S, Boardman LA, Gille JJ, Keller JJ, Westerman AM, Scott RJ, Lim W, Trimbath JD, Giardiello FM, Gruber SB, Offerhaus GJ, de Rooij FW, Wilson JH, Hansmann A, Moslein G, Royer-Pokora B, Vogel T, Phillips RK, Spigelman AD, Houlston RS (2006) Frequency and spectrum of cancers in the Peutz-Jeghers syndrome. Clin Cancer Res 12: 3209-3215

Hemminki A, Markie D, Tomlinson I, Avizienyte E, Roth S, Loukola A, Bignell G, Warren W, Aminoff M, Hoglund P, Jarvinen H, Kristo P, Pelin K, Ridanpaa M, Salovaara R, Toro T, Bodmer W, Olschwang S, Olsen AS, Stratton MR, de la Chapelle A, Aaltonen LA (1998) A serine/ threonine kinase gene defective in Peutz-Jeghers syndrome. Nature 391: $184-187$

Janne PA, Borras AM, Kuang Y, Rogers AM, Joshi VA, Liyanage H, Lindeman N, Lee JC, Halmos B, Maher EA, Distel RJ, Meyerson M, Johnson BE (2006) A rapid and sensitive enzymatic method for epidermal growth factor receptor mutation screening. Clin Cancer Res 12: $751-758$ 
Janne PA, Johnson BE (2006) Effect of epidermal growth factor receptor tyrosine kinase domain mutations on the outcome of patients with nonsmall cell lung cancer treated with epidermal growth factor receptor tyrosine kinase inhibitors. Clin Cancer Res 12: 4416s-4420s

Ji H, Ramsey MR, Hayes DN, Fan C, McNamara K, Kozlowski P, Torrice C, Wu MC, Shimamura T, Perera SA, Liang MC, Cai D, Naumov GN, Bao L, Contreras CM, Li D, Chen L, Krishnamurthy J, Koivunen J, Chirieac LR, Padera RF, Bronson RT, Lindeman NI, Christiani DC, Lin X, Shapiro GI, Janne PA, Johnson BE, Meyerson M, Kwiatkowski DJ, Castrillon DH, Bardeesy N, Sharpless NE, Wong KK (2007) LKB1 modulates lung cancer differentiation and metastasis. Nature 448: $807-810$

Kosaka T, Yatabe Y, Endoh H, Kuwano H, Takahashi T, Mitsudomi T (2004) Mutations of the epidermal growth factor receptor gene in lung cancer: biological and clinical implications. Cancer Res 64: 8919-8923

Launonen V (2005) Mutations in the human LKB1/STK11 gene. Hum Mutat 26: $291-297$

Launonen V, Avizienyte E, Loukola A, Laiho P, Salovaara R, Jarvinen H, Mecklin JP, Oku A, Shimane M, Kim HC, Kim JC, Nezu J, Aaltonen LA (2000) No evidence of Peutz-Jeghers syndrome gene LKB1 involvement in left-sided colorectal carcinomas. Cancer Res 60: 546-548

Marchetti A, Martella C, Felicioni L, Barassi F, Salvatore S, Chella A, Camplese PP, Iarussi T, Mucilli F, Mezzetti A, Cuccurullo F, Sacco R, Buttitta F (2005) EGFR mutations in non-small-cell lung cancer: analysis of a large series of cases and development of a rapid and sensitive method for diagnostic screening with potential implications on pharmacologic treatment. J Clin Oncol 23: 857-865

Matsumoto S, Iwakawa R, Takahashi K, Kohno T, Nakanishi Y, Matsuno Y, Suzuki K, Nakamoto M, Shimizu E, Minna JD, Yokota J (2007) Prevalence and specificity of LKB1 genetic alterations in lung cancers. Oncogene 26: $5911-5918$

Naoki K, Chen TH, Richards WG, Sugarbaker DJ, Meyerson M (2002) Missense mutations of the BRAF gene in human lung adenocarcinoma. Cancer Res 62: $7001-7003$
Ono M, Hirata A, Kometani T, Miyagawa M, Ueda S, Kinoshita H, Fujii T, Kuwano M (2004) Sensitivity to gefitinib (Iressa, ZD1839) in non-small cell lung cancer cell lines correlates with dependence on the epidermal growth factor (EGF) receptor/extracellular signal-regulated kinase 1/2 and EGF receptor/Akt pathway for proliferation. Mol Cancer Ther 3: $465-472$

Onozato R, Kosaka T, Achiwa H, Kuwano H, Takahashi T, Yatabe Y, Mitsudomi T (2007) LKB1 gene mutations in Japanese lung cancer patients. Cancer Sci 98: 174-175

Pham D, Kris MG, Riely GJ, Sarkaria IS, McDonough T, Chuai S, Venkatraman ES, Miller VA, Ladanyi M, Pao W, Wilson RK, Singh B, Rusch VW (2006) Use of cigarette-smoking history to estimate the likelihood of mutations in epidermal growth factor receptor gene exons 19 and 21 in lung adenocarcinomas. J Clin Oncol 24: $1700-1704$

Sanchez-Cespedes M, Parrella P, Esteller M, Nomoto S, Trink B, Engles JM, Westra WH, Herman JG, Sidransky D (2002) Inactivation of LKB1/STK11 is a common event in adenocarcinomas of the lung. Cancer Res 62 $3659-3662$

Shaw RJ, Bardeesy N, Manning BD, Lopez L, Kosmatka M, DePinho RA, Cantley LC (2004) The LKB1 tumor suppressor negatively regulates mTOR signaling. Cancer Cell 6: $91-99$

Tiainen M, Ylikorkala A, Makela TP (1999) Growth suppression by Lkb1 is mediated by a G(1) cell cycle arrest. Proc Natl Acad Sci USA 96: 9248-9251

Tracy S, Mukohara T, Hansen M, Meyerson M, Johnson BE, Janne PA (2004) Gefitinib induces apoptosis in the EGFRL858R non-small-cell lung cancer cell line H3255. Cancer Res 64: $7241-7244$

Volikos E, Robinson J, Aittomaki K, Mecklin JP, Jarvinen H, Westerman AM, de Rooji FW, Vogel T, Moeslein G, Launonen V, Tomlinson IP, Silver AR, Aaltonen LA (2006) LKB1 exonic and whole gene deletions are a common cause of Peutz-Jeghers syndrome. J Med Genet 43: e18 